\title{
Mobile Virtual Network Operator Strategy for Migration towards 4G
}

\author{
Yacouba Coulibaly*, Georges Kaddoum*, and Mohammed F. A. Ahmed *† \\ *University of Québec, École de Technologie Supérieure, Montréal, Canada \\ $\dagger$ Electrical Engineering Department, Assiut University, Assiut, Egypt \\ Email: yacouba.coulibaly.4@ens.etsmtl.ca, georges.kaddoum@etsmtl.ca,m.ahmed@ieee.org
}

\begin{abstract}
Recently, mobile network operators (MNOs) around the world are migrating their networks to the fourth generation (4G) long-term evolution (LTE) standard. Mobile virtual network operator (MVNOs), who do not have their own wireless access network and still offer added value services, are affected with this migration. Most of the research conducted about network migration toward the $4 \mathrm{G}$ focused on challenges in the architecture level and the business model. Also, research addressed the compatibility of core networks with $4 \mathrm{G}$, and the interfaces and protocols that must be implemented by the MVNO to continue offering its services. This paper proposes different approach by focusing on the prospects of services. Typically, the service cost should decline with the arrival of the new 4G network. We suggest some strategies that could be adopted by MVNOs to offer more competitive services and contribute in reducing the costs of communication of customers.
\end{abstract}

Keywords-Mobile virtual network operator (MVNOs), fourth generation (4G) Migration, and long-term evolution (LTE).

\section{INTRODUCTION}

One of the most important projects in recent years among mobile network operators (MNOs) is the network migration to long-term evolution (LTE). The new network is expected to eliminate the distinction between voice and data services as all services use data protocols. The new network is expected to provide an improvement in bandwidth and throughput as compared to its predecessors. However, while the network of some MNOs already changed to $4 \mathrm{G}$, the cost for the end user has not decreased.

Mobile virtual network operator (MVNOs) are using the same access network with MNOs by renting minutes and data volume wholesale for resale to their customers. Therefore, it is trying to comply with the migration to $4 \mathrm{G}$ network and offer $4 \mathrm{G}$ services. Previous research in this area was oriented to architecture issues, namely the possible business model for MVNOs and compatibility of the $4 \mathrm{G}$ networks with the core network of MVNOs. This paper proposes a strategy for the added value services to help reducing the cost of international roaming and other services for the end user. The rest of the paper is organized as follows. Section II defines the value chain of MVNOs. Section III presents the state of the art in mobile networks and Section IV shows the service cost of the current technology. Section $\mathrm{V}$ addresses the challenges facing MVNOs. Section VI addresses the radio spectrum sharing and Section VII proposes some strategies for the services that can make MVNOs more competitive. Finally, Section VIII presents conclusions and future work.

\section{VALUE CHAIN IN MVNOS}

An MVNO is a mobile service provider that does not have its own mobile radio license (access network). It uses the network resources of a MNO and buys wholesale services (i.e. minutes of voice, volume of data, SMS and others). It potentially provides all other elements in the value chain as shown in Fig. 1. From the perspective of the customer, the MVNO delivers all the services [1]. There are different business models for the MVNOs which depend on how the value chain is restructured, namely,

- $\quad$ Branded reseller: The lightest business model, MVNO just provides its brand and its distribution channels.

- Light-MVNO: An intermediate business model between a branded reseller and a full-MVNO.

- Full-MVNO: The complete business model, where the MNO provides the access network infrastructure, while the MVNO provides the rest of the elements of the value chain. It allows complete control of the infrastructure. This model is adopted by many operators.

- Mobile virtual network enablers (MVNE): This is a provider of infrastructure that facilitates the launch of MVNO operations. An MVNE can be positioned between the host MNO and the MVNO. MVNEs reduce the entry barriers for the MVNOs.

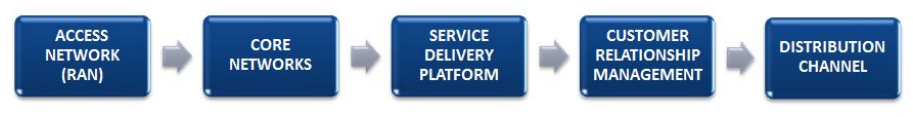

Fig. 1. Mobile value chain [1], [2]

In the following, we only discuss the case of the fullMVNO. Because this model is flexible, it allows to take control of infrastructure and become independent. It is the recommended model for an MVNO. With This model, MVNO can participate in all of or part of the mobile value chain (excepting access network) as shown in Fig. 1.

\section{STATE OF THE ART OF THE MOBILE ACCESS NETWORKS}

LTE is a 3GPP project to evolve the UMTS technology towards $4 \mathrm{G}$ mobile network. Namely, the LTE standards are defined in 3GPP release 8 specifications. The 4G technology has a lot of advantages like enhanced user experience, high 


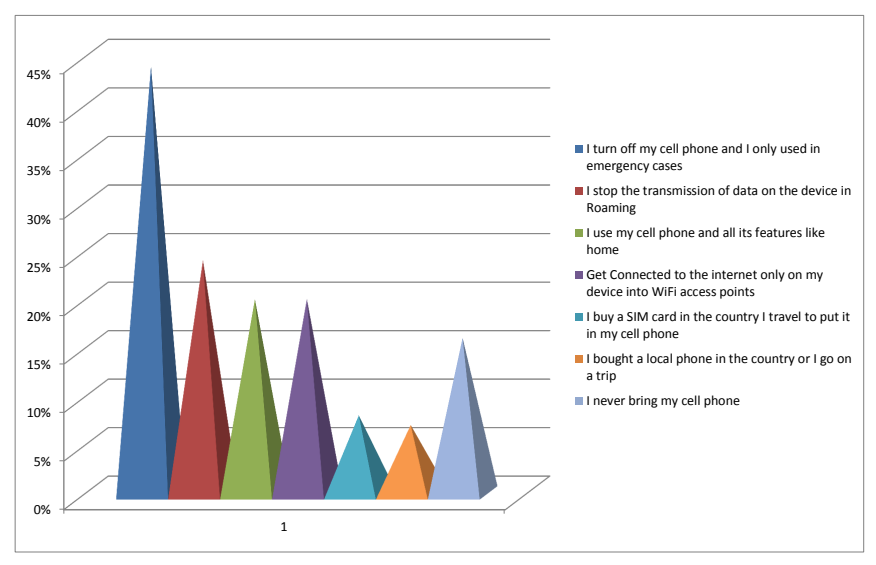

Fig. 2. Survey using phone international roaming

broadband mobile technology, simplified network architecture (IP-based), high level of security, robust Quality of service (QoS) framework, and common evolution for multiple technologies. The LTE is expected to reduce the communication cost relatively. This technology is "all IP" and would replace the previous technologies which was based on circuit switching. In circuit switching, each user occupies a specific circuit for the whole duration of the communication which consequently results in high cost communication.

\section{High COSTS OF THE COMMUNiCATIONS}

A report by the organisation for economic co-operation and development (OECD) compared the prices of wireless data roaming among OECD countries. This report ranked Canada as the first country in the OECD countries for the cost associated with transmission of roaming data for single exchange session traffic of $1 \mathrm{Mb}$. The OECD method was to examine the retail roaming prices for data transmission charged to subscribers by the two largest mobile operators in each of the 34 OECD countries [3]. Public interest advocacy centre of Canada (PIAC) has also conducted a survey on roaming of the Canadians abroad. The survey has asked this question: "Which statement best describes what you usually do with your cell phone when you leave Canada on a private trip?" The survey results are shown in Fig. 2. We see in this study that the largest percentage of people (44\%) turn off their cell phones and only use it in emergency cases. A small percentage $(8 \%)$ bought a local phone with SIM card in the country they are travelling to. Also, $24 \%$ of them stop the data transmission on the device in roaming. $20 \%$ use cell phone and all its features the same way as home while prefer to connected to the internet only through Wi-Fi access point. And at the end, the group of people who do not take cell phones while travelling abroad. This study attributes this high price to a lack of transparency and competition. This behaviour of people is an alarm of the high price of this service by the operators. The European commission in a study in 2006 found revenue margins from $300 \%$ to $400 \%$ for the operators. This report concluded that the expenses of roaming were difficult to prove and that the prices of roaming abroad were raised without justification.

\section{Challenges to Face}

\section{A. MVNO Business model}

One of the contributing factors to the success of an MVNO is its ability to control its infrastructure or the level of resources it uses to provide services. Namely, databases, billing, roaming, control the SIM card of the customers, implementing its own policy (monitoring, customers service delivery) and its interconnection to other networks. We can observe different types of the full-MVNOs [4], [5]:

- $\quad$ Simple Full-MVNO model: The MVNO install its own core equipment and connected to one host MNO.

- Multi-MNO model: The MVNO connects to several MNOs.

- $\quad$ Always-best-connected model: The MVNO can select automatically between several MNOs according to certain criteria.

\section{B. Migration of full-MVNO toward $4 G$}

The choice of the business model of MNVO will impact more or less the core network infrastructure for several reasons including: 2G/3G of MVNO networks are not fully compatible with LTE; Core network signalling has moved from traditional MAP, SS7 and RADIUS protocols to all-Diameter signalling protocol in LTE and other proprietary interfaces (with other networks). Migration of full-MVNO 2G/3G toward 4G needs to mitigate different issues [6]: Ability to introduce services independently; interworking and inter-administration interfaces; diameter signalling controller (DSC); enforcing Policy through another network/access; detecting user behaviour and context data; selecting best alternative access network.

\section{Enhanced packet core (EPC) network}

MVNO operates with small revenue margins, consequently, upgrading all network infrastructure to $4 \mathrm{G}$ can be very expensive in the capital expenditure (CAPEX). However, inevitable investment is required to enjoy the benefits offered by the LTE. In this case, it can be recommended that MVNO chooses multiMNO with always-best-connected model, as shown in Fig. 3, to have flexibility and control of its infrastructure and futures services. Both of the following strategies are suggested for core network infrastructure acquisition:

- First, MVNO makes an important investment in CAPEX for its core network like [7]: IMS, update old HLR to HSS, serving gateway (S-GW), PCRF to enforce policy, proxy $3 \mathrm{GPP}$ AAA server, PDG, online charging system (OCS), and other. This can permit the interworking of $2 \mathrm{G} / 3 \mathrm{G}$ infrastructure with 4G. The ROI of this strategy can be very long but still allow MVNOs to provide $4 \mathrm{G}$ services to subscribers. Due to the long implementation time of core network elements, this strategy is expensive and does not allow MVNO to focus on the development of the services.

- $\quad$ Second, using a service level agreement (SLA), face of intense competition, MVNO should avoid heavy capital investment and search for more flexible solutions such as network sharing. This could be done 


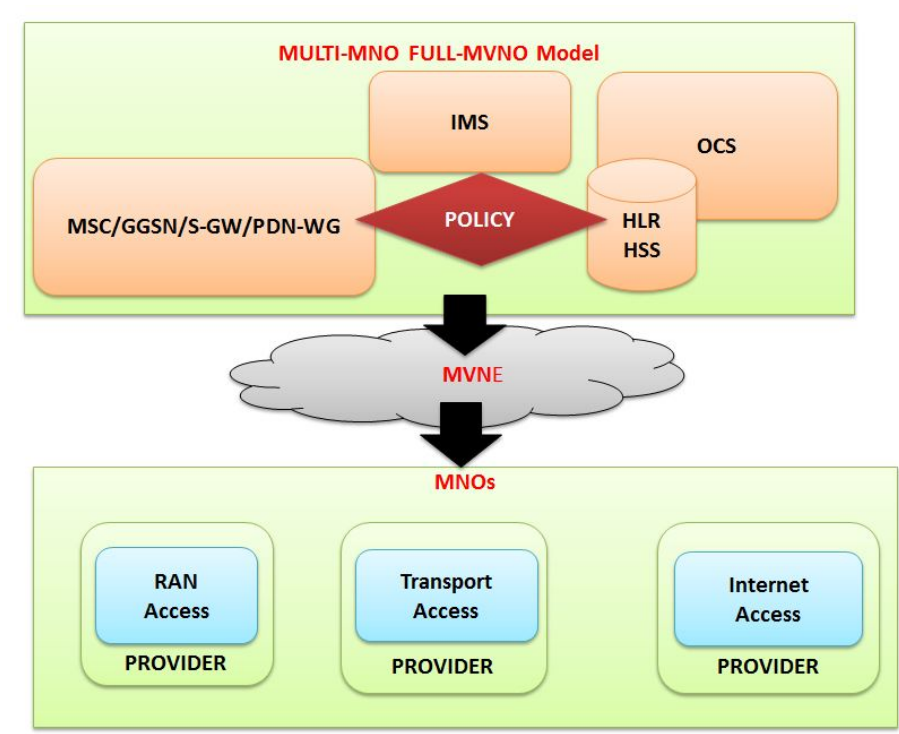

Fig. 3. Multi-MNO Full-MVNO model

through an SLA with mobile virtual network enabler (MVNE) [8] or mobile network aggregator (MNA). This strategy is more flexible in CAPEX and saves implementation time while having faster ROI. Moreover, it allows MVNOs to focus on added value services development.

\section{MVNO RADIO SPECTRUM SHARING}

One of the important elements that MVNOs should check with their host MNO is the effective sharing scheme used by the MNO to share the radio spectrum between its MVNOs. This is capital for access to services for MVNO subscribers. There are several methods of radio spectrum sharing [8]. Fixed spectrum allocation scheme (FSA) is simple but it has the lowest level of sharing and the lowest spectral efficiency and, accordingly, waste of spectrum resource. Partial spectrum sharing scheme (PSS) promote spectral efficiency, but spectral utilization rate is still low. Dynamic spectrum sharing scheme (DSS) is based on dynamic prioritization of MVNOs, no fairness, and can not be guarantee due to insensitive priority factor. All of the three schemes can not guarantee full usage of radio spectrum and the basic interest of MVNOs. In order to protect MVNOs interest, a based multi-operator shared network opportunistic spectrum sharing (BMSN-OSS) [8] is a good choice for MVNOs. This scheme can permit UEs of MVNOs to use the unoccupied spectrum among them.

\section{Proposed StRategy OF ACtivities}

Based on the OECD study [3], we can say that reducing the price of international roaming services is still a huge challenge. We believe that it is a consequence of the low competition in Canada among the operators in the mobile network field. There is also a phenomenon known as the bill shock where the operator charges unexpectedly huge bill for roaming. Given the current situation, we believe that the MVNO in North America, particularly in Canada, have a potential market in this sector. But to do this, MVNOs have to be very innovative and opportunistic. To overcome this challenge, MVNOs should be equipped with infrastructure to: Control of roaming customers, develop a platform to exchange control of machine to machine (M2M) data, develop over the top (OTT) applications, provide content services, implement measures to protect the customers, and deal with a MNO who use a best scheme to sharing radio spectrum resources in its access network. With that MVNOs should be very competitive at the level of services to reduce the cost and also to gain parts of the market.

\section{A. International roaming rates}

MVNE offers services to aggregate networks and provides a complete solution to full MVNO like core network, back office services. Specifically, MVNE offers the following services [8]: Provide infrastructure to support multiple MVNOs; Offer an access to several roaming partners(national and international); Provide real-time adaptation network functions and needs in terms of performance; A use of services as required MVNO; Quote a rate per use. Strategically an MVNE is an important partner for the MVNO [9] to acquire infrastructure in a relatively short time and saves significant investments. This would allow the MVNO to focus on what its chosen field. As shown in Fig. 3 [5], the MVNO can make a flexible price for the roaming service to the customers. With competitive tariffs, MVNOs could profit of high price of roaming in the world by offering competitive services like voice, data, OTT applications. As Google talk phone which is limited to voice communication to the north America, the similar applications can be develop by MVNOs and be use everywhere.

\section{B. $M 2 M$}

$\mathrm{M} 2 \mathrm{M}$ is the communication between machines including virtual automated data exchange. M2M communications based on mobile network technology is a new opportunities for MVNO. Many services can be offered like: Remote management, Security \& Surveillance, Fleet Management, e-Health Applications, Sales \& Payment, Home Applications. For instance, MVNOs could provide interesting solutions to the automotive industry which has the following limitations and requirements [10]: Higher traffic control: to address different service requirements and have more network control; Best possible coverage: for the best user experience and higher service reliability; SIM cards independent from MNO; Standardized interconnect to MNOs: to manage multiple MNOs procedures and reduce the operating complexity; Control/Elimination of roaming fees: to equalize the user service experience in all the countries, independent of where the car is manufactured, where it is sold, and where it is driven. These different problems can be mitigated by MVNOs and can extend their market to the automotive sector [5], [8]-[10].

\section{OTT applications}

Since apparution in the environment of mobile network, MVNO have always innovated. But it should use the right tools like service-oriented architecture (SOA), for service development. SOA is a designing, developing, deploying and managing systems characterized by reusable functionality with standard interfaces. Adopting SOA provides cost-efficiency, business agility, adaptability and leverage of legacy systems [11]. This platform can allow developing variable applications to offer several services. Integrated application as "facetime", "i-messenger", "Skype", "Google talk phone" which offer 


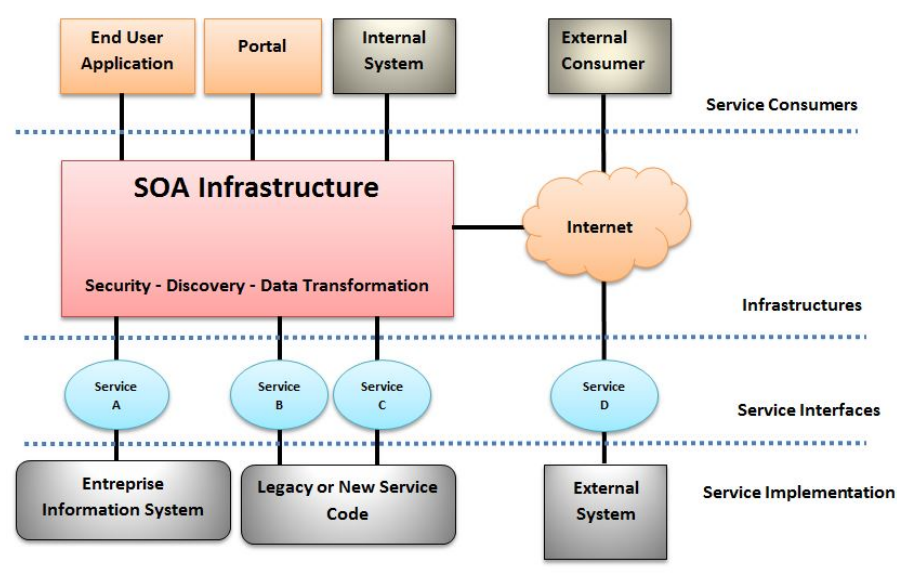

Fig. 4. High-level representation of a service oriented System [11]

voice, data and video communications and rich user interface experience to exploit the low cost of data transportation as shown in Fig. 4. The penetration of OTT applications on mobile phones is still low. Many initiatives start to prove itself in the market of services. For instance Apple contemplates SIMs software in the smartphones without operator control. The OTT application development is a potentially huge market for the MVNOs which requires the integration of hardware and software. According to the Google/Android initiative of developing an OS that can run on all mobile devices, it is important and interesting to cover large part of the market [12].

\section{Content services}

MVNOs can be more agile and more inventive and monetize the information they have about the users. For example, following the habits of their users and selling that information to advertisers provide dynamic ad insertion and individualize the ads according to each users profile. This market begins to expand for services and MVNOs should take part of it.

\section{CONCLUSIONS AND FUTURE WORK}

In this paper, the challenges of LTE migration for MVNO have been studied. The business model and strategy allow MVNO to access an independence from the host MNO. This includes investing CAPEX in the network infrastructure depending on the adopted strategy. For multi-MNO with alwaysbest-connected model, MVNOs have to focus on the creation of added value in order to be competitive on the market. To do this, MVNOs must use MVNE services to access rapidly to the infrastructures without having to deploy more CAPEX and take more risk. The availability of smart devices opens a new market to the MVNOs which can increase significantly their value chain. The strategy to mitigate the CAPEX of the business models gives advantages to MVNO like flexibility and large proposed services. Additionally, the migration toward $4 \mathrm{G}$ brings many advantages to MVNO. In the future, when cloud computing becomes applicable to the telecommunication network, the network elements could be implemented in clouds, operators could utilize all kinds of cloud computing services, like [13] Infrastructure (IaaS), Platform (PaaS) and Software (SaaS) as a Service. Operators with most interest in cloud computing would be the MVNOs because their CAPEX and exploitation (OPEX) will become easier, faster and more efficient [8].

\section{REFERENCES}

[1] M. Balon and B. Liau, "Mobile virtual network operator," in Telecommunications Network Strategy and Planning Symposium (NETWORKS), 2012 XVth International, Oct. 2012, pp. 1 - 6.

[2] K. Pousttchi and Y. Hufenbach, "Analyzing and categorization of the business model of virtual operators," in Mobile Business, 2009. ICMB 2009. Eighth International Conference on, Jun. 2009, pp. 87 - 92.

[3] J. Bishop and J. Lo, "Consumers and wireless data roaming," Public Interest Advocacy Centre, Tech. Rep., 2012.

[4] S. Pattanavichai and W. Premchaiswadi, "Pricing models for MVNE in supporting MVNO investment in 3G UMTS network," in Knowledge Engineering, 2010 8th International Conference on ICT and, Nov. 2010, pp. $82-88$.

[5] R. Copeland and N. Crespi, "Modelling multi-MNO business for MVNOs in their evolution to LTE, VoLTE \& advanced policy," in BMMP'11 : Third International Workshop on Business Models for Mobile Platforms : Access and Competitiveness in Multi-Sided Markets, IEEE, Ed., 2011, pp. 295 - 300.

[6] _ " "Resolving ten MVNO issues with EPS architecture, VoLTE and advanced policy server," in ICIN'11 : 15th International Conference on Intelligence in Next Generation Networks : From Bits to Data, from Pipes to Clouds, 2011, pp. $29-34$

[7] S. Pattanavichai, N. Jongsawat, and W. Premchaiswadi, "Real options analysis for valuing strategic investments and decisions of the mobile virtual network operator's investment in E-UMTS," in ICT and Knowledge Engineering (ICT Knowledge Engineering), 2011 9th International Conference on, Jan. 2012, pp. $138-144$.

[8] P. Wang, B. Wang, W. Wang, Y. Zhang, and C. Wang, "Based multioperator shared network opportunistic spectrum sharing," in Cloud Computing and Intelligent Systems (CCIS), 2012 IEEE 2nd International Conference on, vol. 2, Oct. 2012, pp. $864-868$

[9] M. De Leon and A. Adhikari, "A user centric always best connected service business model for mvnos," in Intelligence in Next Generation Networks (ICIN), 2010 14th International Conference on, Oct. 2010, pp. $1-8$.

[10] C. Machuca, T. Krauss, A. Basta, E. Grigoreva, and W. Kellerer, "Alternatives for the automotive industry to become an MVNO," in Transparent Optical Networks (ICTON), 2014 16th International Conference on, Jul. 2014, pp. 1 - 4.

[11] G. A. Lewis and D. B. Smith, "Service-oriented architecture and its implications for software maintenance and evolution," in Frontiers of Software Maintenance, 2008. FoSM 2008., Sep. 2008, pp. 1 - 10.

[12] A. Hammershoj, A. Sapuppo, and R. Tadayoni, "Challenges for mobile application development," in Intelligence in Next Generation Networks (ICIN), 2010 14th International Conference on, Oct. 2010, pp. 1 - 8.

[13] R. Yrjo and D. Rushil, "Cloud computing in mobile networks - case MVNO," in Intelligence in Next Generation Networks (ICIN), 2011 15th International Conference on, Oct. 2011, pp. 253 - 258. 